\title{
Effect of Cysteine Derivatives Administration in Healthy Men Exposed to Intense Resistance Exercise by Evaluation of Pro-Antioxidant Ratio
}

\author{
Agnieszka ZEMBRON-LACNY ${ }^{1}$, Kazimierz SZYSZKA ${ }^{1}$, and Zbigniew SZYGULA ${ }^{2}$ \\ ${ }^{1}$ Department of Sport Medical Sciences, Faculty of Physical Culture, University of Physical Education Poznan, Gorzow Wlkp., Poland; \\ and ${ }^{2}$ Department of Sport Medicine, University of Physical Education Krakow, Poland
}

\begin{abstract}
The aim of this study was to ascertain the influence of cysteine derivatives on pro-antioxidant equilibrium and to compare the antioxidant effectiveness of $\mathrm{N}$-acetylcysteine, $\alpha$-lipoic acid, and taurine by using Loverro's coefficient (pro-antioxidant ratio) in healthy men exposed to intensity-resistance exercise. Fifty-five men were randomly assigned to one of four groups: control (CON, placebo), $\mathrm{N}$-acetylcysteine (NAC $1.8 \mathrm{~g}^{-1 a y}{ }^{-1}, 3$ days), $\alpha$-lipoic acid (LIP $1.2 \mathrm{~g}$ day-1 3 days), or taurine (TAU 3 $\mathrm{g} \cdot$ day $^{-1}, 3$ days). The erythrocyte superoxide dismutase (SOD), glutathione peroxidase (GPx), and catalase (CAT) activities, lipid peroxidation products (TBARS), and plasma protein thiol concentrations were evaluated. The P/A ratio was determined from the mean values of TBARS, SOD, GPx, and CAT. The applied exercise at maximal intensity induced the significant changes in
\end{abstract}

pro-antioxidant equilibrium toward peroxidation, which was proved by a $25 \%$ increase in TBARS concentration in the CON group. The peroxidation was significantly diminished by NAC $(-14 \%)$ and LIP $(-16 \%)$, whereas TAU had no effect on the TBARS concentration. Cysteine derivatives administration prevented exercise-induced decline in SOD activity and increased in GPx activity during exercise. CAT activity changed only in the LIP group. The estimation of P/A ratio showed the lowest level of pro-antioxidant equilibrium after LIP administration. In the CON group, P/A ratio was directly correlated with the protein thiols level $(r=0.495, p<0.001)$. These data confirm the antioxidant action of tested cysteine derivatives, particularly lipoic acid, and demonstrate the practical application of P/A ratio to evaluate the effectiveness of antioxidants in athletes.

Key words: oxidative stress, $N$-acetylcysteine, $\alpha$-lipoic acid, taurine, athletes.

$E_{x}$ tive oxygen species (ROS) generation and antioxidant depletion, and consequently the shift in pro-antioxidant equilibrium toward the peroxidation that can lead to negative changes in cell metabolism. These changes include the inhibition of enzymes and membrane receptor activities, the release of proinflammatory cytokines, an increase in $\mathrm{Ca}^{2+}$ concentration, and an activation of proteases and nucleases, as well as structural cell damage [1].

The intervention consisting in the weakening of ROS generation by antioxidant supplementation may prevent the adverse effects of exercise. Nevertheless, as shown in several studies the use of antioxidant vitamins hindered muscle repair after exercise, whereas the additional intake of sulfur-containing compounds could increase the defense against ROS $[2,3]$.

$\mathrm{N}$-Acetylcysteine (NAC) is probably the most effective thiol compound. The studies showed that oral, intravenous or intraperitonal supplementation of NAC prevented a decline in muscle, lung, and blood thiols level and weakened exercise-induced glutathione oxidation. Besides the stimulation of glutathione synthesis, NAC also reduced extracellular cystine to cysteine, directly affected ROS, increased erythropoietin level, and also regulated the activities of catalase, mitochondrial superoxide dismutase, glutathione $S$-transferase, and different forms of glutathione peroxidase, eventually resulting in a significant preservation of membrane lipid $[1,3]$.

$\alpha$-Lipoic acid and its reduced form dihydrolipoate directly react with ROS and protect membranes by interacting with vitamin $\mathrm{C}$, vitamin $\mathrm{E}$, and glutathione. Moreover, as lipoamid, $\alpha$-lipoic acid functions as a cofactor in the multienzyme complexes that catalyse the oxidative decarboxylation of $\alpha$-keto acids, such as pyruvate, $\alpha$-ketoglutarate, and branched chain $\alpha$-keto acids [3, 4]. $\alpha$-Lipoic acid is commonly used as a dietary supplement by athletes to increase muscle glucose uptake, improve antioxidant defense, and decrease oxidative damage level. However, most studies concerning the beneficial effects of $\alpha$-lipoic acid on cell metabolism were observed in in vitro studies or animal models, such as rodents and horses [5-9].

Received on Sep 2, 2007; accepted on Nov 12, 2007; released online on Nov 15, 2007; doi:10.2170/physiolsci.RP009307 Correspondence should be addressed to: Agnieszka Zembron-Lacny, Department of Sport Medical Sciences, Faculty of Physical Culture, University of Physical Education Poznan, ul. Estkowskiego 13, 66-400 Gorzow Wlkp., Poland. E-mail: agzem@gorzow.home.pl 


\section{A. ZEMBRON-LACNY et al.}

Taurine (2-aminoethanesulfonic acid) is an amino acid derived from cysteine metabolism and is involved in cell volume regulation, antioxidation, detoxification, and carbohydrate metabolism. For this reason, it has been commonly included in sport nutritional supplements. In contrast to $N$-acetylcysteine and $\alpha$-lipoic acid, there is less evidence supporting the potential antioxidant benefits of taurine supplementation [10-12].

The aim of this study was to ascertain the influence of three-day oral treatment with cysteine derivatives on proantioxidant equilibrium and to compare the antioxidant effects of $N$-acetylcysteine, $\alpha$-lipoic acid, and taurine by using Loverro's pro-antioxidant coefficient in healthy men exposed to systemic eccentric exercise.

\section{METHODS}

Fifty-five healthy trained young males (canoeists and rowers, Table 1), physical education students, participated in a randomized and placebo-controlled study. The subjects had used no antioxidant supplements (vitamins or minerals) for 4 weeks prior to the study. They were not allowed to exercise for $48 \mathrm{~h}$ before and $24 \mathrm{~h}$ after the exercise test. All the subjects were informed of the aim of the study and gave their written consent for participation in the research project. The protocol of the study was approved by the Local Bioethical Committee in Gorzow Wlkp in accordance with the Helsinki Declaration of 1975, as revised in 1983 (41st World Medical Assembly Declaration of Helsinki, 1990).

We administered $1.8 \mathrm{~g} \cdot$ day $^{-1}$ of $N$-acetylcysteine (NAC group, Hexal AG, Germany), $1.2 \mathrm{~g} \cdot$ day $^{-1}$ of $\alpha$-lipoic acid (LIP group, Wörwag Pharma, Germany), $3 \mathrm{~g} \cdot \mathrm{day}^{-1}$ of taurine (TAU group, Olimp Sport Nutrition, Poland), or a placebo $3 \times 0.35 \mathrm{~g} \cdot \mathrm{day}^{-1}$ of saccharum lactis (CON group) for three days in three doses as powder dissolved in $50 \mathrm{ml}$ water. The subjects took the first dose in the morning in a fasted state, the second dose at noon, and the final dose $2 \mathrm{~h}$ before evening training.

The subjects performed an intensity-resistance exercise (strength test). The resistance-exercise protocol consisted of a shoulder press, bench press, and dead lift executed in series. This means that each exercise element was performed five times with no breaks; then after a 1-min break, the series was repeated. The subjects performed from 5 to 7 series. The initial load was $50 \% 1 \mathrm{RM}(60 \mathrm{~kg})$, then increased by $10 \mathrm{~kg}$ after each series of exercise elements, until exhaustion. The $120-\mathrm{kg}$ weight lifted by athletes during training was determined as maximal load, i.e., 1 RM. Before the test, the athletes carried out individual warm-ups with no elements of strength exercise.

Blood samples were obtained from a cubital vein with an anticoagulant $\left(\mathrm{EDTAK}_{2}\right)$ before exercise, immediately after completing the exercise, and after a $24 \mathrm{~h}$ rest. The samples were immediately placed in $4^{\circ} \mathrm{C}$ temperature after collection. Within $10 \mathrm{~min}$, the blood samples were centrifuged $\left(2,500 \times g, 10 \mathrm{~min}, 4^{\circ} \mathrm{C}\right)$. The aliquots of plasma were stored at $-20^{\circ} \mathrm{C}$. Erythrocyte fraction was resuspended three times in cold isotonic saline solution and centrifuged $\left(2,500 \times g, 10 \mathrm{~min}, 4^{\circ} \mathrm{C}\right)$. Washed erythrocytes were stored at $-20^{\circ} \mathrm{C}$ until analysis. All samples were analyzed within 7 days.

Protein thiols. The plasma protein thiol concentration was estimated by the method of Hebeeb [13], using dithionitrobenzene (DTNB, Sigma, Germany). The samples were added to a denaturing solution containing sodium dodecyl sulfate to ionize the sulfhydryl groups, thus making them more reactive to DTNB. The samples were measured at $410 \mathrm{~nm}$ against the control sample (minus DTNB). The intra-assay coefficient of variation (CV) for thiol procedures was $<10 \%$.

Antioxidant enzymes. In the erythrocytes, the activities of superoxide dismutase (SOD, EC 1.15.1.1) and glutathione peroxidase (GPx, EC 1.11.1.9) were evaluated using Randox kits (UK). GPx activity was assayed in an enzyme-coupled reaction by detecting the changes in the NADPH level. Cumene hydroperoxide were used as substrates. It was originally described by Paglia and Valentine [14]. SOD activity was measured by the degree of inhibition of xanthine and xanthine oxidase reaction, which generate superoxide radicals. SOD and GPx detection limits for Randox kits were $0.06 \mathrm{U} \cdot l^{-1}$ and $8.86 \mathrm{U} \cdot l^{-1}$, respectively. The intra-assay coefficient of variation $(\mathrm{CV})$ for the SOD kit was $8.13 \%$, and for the GPx kit $4.20 \%$.

Erythrocyte catalase (CAT, EC 1.11.1.6) was measured by the method of Aebi [15], using the first order rate con-

Table 1. The characteristics of the subjects: control group (CON) and experimental groups (NAC, LIP, TAU).

\begin{tabular}{ccccccc}
\hline \multirow{2}{*}{ Group } & Age (years) & Height $(\mathrm{cm})$ & Body mass $(\mathrm{kg})$ & Body fat $(\%)$ & \multicolumn{2}{c}{ Lactate $\left(\mathrm{mmol} \cdot r^{-1}\right)$} \\
\cline { 5 - 7 } & & & & & Pre-exercise & Post-exercise \\
\hline CON $(n=15)$ & $21.5 \pm 1.4$ & $181.7 \pm 8.3$ & $87.2 \pm 10.6$ & $14.4 \pm 4.6$ & $1.40 \pm 0.33$ & $8.54 \pm 1.78^{* *}$ \\
NAC $(n=15)$ & $21.9 \pm 1.7$ & $180.7 \pm 7.4$ & $87.1 \pm 12.8$ & $14.5 \pm 5.6$ & $1.61 \pm 0.39$ & $8.57 \pm 1.92^{* *}$ \\
LIP $(n=10)$ & $22.8 \pm 1.0$ & $183.7 \pm 7.9$ & $86.9 \pm 14.0$ & $14.1 \pm 4.9$ & $1.89 \pm 0.55$ & $8.77 \pm 3.30^{* *}$ \\
TAU $(n=15)$ & $21.9 \pm 1.3$ & $177.1 \pm 7.6$ & $82.1 \pm 10.7$ & $12.3 \pm 5.2$ & $1.76 \pm 0.43$ & $8.03 \pm 1.67^{* *}$ \\
\hline
\end{tabular}

${ }^{*} p<0.05$ and ${ }^{* *} p<0.01$ are significantly different from the pre-exercise value. 
stant of the decomposition of hydrogen peroxide by erythrocyte catalase.

Marker of oxidative damage. Erythrocyte lipid peroxidation products were estimated using the measurement of thiobarbituric acid-reactive substance (TBARS) level according to the method of Buege and Aust [16]. We added $500 \mu \mathrm{l}$ trichloroacetic acid to $25 \mu \mathrm{l}$ erythrocytes and mixed. Then $500 \mu$ thiobarbituric acid was added, mixed, and incubated at $100^{\circ} \mathrm{C}$ for $10 \mathrm{~min}$ to prevent an exogenous amplification of peroxidation. After cooling, the precipitate is removed by centrifugation at $1,000 \times g$ for 20 min. The samples were measured at $535 \mathrm{~nm}$ against control samples with no thiobarbituric acid. The TBARS level was expressed as nmol of malondialdehyde using 1,1,3,3-tetraethoxypropane as standard (Fluka, Germany). TBARS detection limit for the method was $0.13 \mathrm{nmol} \cdot \mathrm{ml}^{-1}$. The intra-assay coefficients of variation $(\mathrm{CV})$ for TBARS procedures were $<10 \%$.

All the results in enzymatic activities and peroxidation products concentration were expressed relatively to $\mathrm{Hb}$ concentration measured by the Drabkin method [17], using Drabkin's reagent (POCH, Poland).

The pro-antioxidant $(\mathrm{P} / \mathrm{A})$ ratio was determined from the mean values of TBARS, SOD, GPx, and CAT according to Loverro et al. [18]:

\section{$\mathrm{P} / \mathrm{A}=\mathrm{TBARS} /(\mathrm{SOD}+\mathrm{GPx}+\mathrm{CAT})$}

The lactate (LA) concentration in the whole blood was assessed using a Dr. Lange kit (Germany).

Statistics. To determine the effects of exercise and supplements, a statistical analysis was performed using twoway ANOVA and a post hoc Newman-Keul's test. The correlations between observed parameters were analyzed by the Pearson correlation coefficient. Statistical significance was set at $p<0.05$. Data are presented as mean \pm SD.

\section{RESULTS}

The applied exercise at maximal intensity (post-exercise lactate: CON $8.54 \pm 1.78 \mathrm{mmol} \cdot l^{-1}$, NAC $8.57 \pm 1.92$ $\mathrm{mmol} \cdot l^{-1}$, LIP $8.77 \pm 3.30 \mathrm{mmol} \cdot l^{-1}$, TAU $8.03 \pm 1.67$ $\mathrm{mmol} \cdot l^{-1}$ ) caused significant changes in tested parameters. The results of the present study are shown in Tables $1-3$.

The concentration of plasma protein thiols significantly increased by $13-25 \%$ immediately after a strength test in each group. A three-day treatment with NAC and LIP significantly elevated the concentration of protein thiols more than $13 \%$ at rest, whereas TAU supplementation induced no change in their level.

Table 2. Levels of parameters of pro-antioxidant equilibrium.

\begin{tabular}{|c|c|c|c|c|c|c|}
\hline Parameter & Pre-exercise & Supp. vs. CON & Post-exercise & Supp. vs. CON & Rest $24 \mathrm{~h}$ & Supp. vs. CON \\
\hline \multicolumn{7}{|c|}{ Protein thiols $\mathrm{mg} \cdot \digamma^{-1}$} \\
\hline CON & $110.13 \pm 9.43$ & & $134.74 \pm 12.39^{*}$ & & $114.60 \pm 19.46$ & \\
\hline NAC & $124.42 \pm 7.53$ & 0.023 & $145.03 \pm 24.17^{\star}$ & ns & $124.42 \pm 24.09$ & ns \\
\hline LIP & $127.70 \pm 5.76$ & 0.014 & $144.50 \pm 20.50^{\star *}$ & ns & $110.00 \pm 17.58$ & ns \\
\hline TAU & $103.65 \pm 9.72$ & ns & $129.06 \pm 8.27^{*}$ & ns & $107.73 \pm 10.70$ & ns \\
\hline \multicolumn{7}{|c|}{ TBARS $n m o l \cdot g^{-1} \mathrm{Hb}$} \\
\hline CON & $111.00 \pm 18.94$ & & $138.34 \pm 21.48^{\star *}$ & & $96.52 \pm 11.81$ & \\
\hline NAC & $95.90 \pm 14.93$ & ns & $118.50 \pm 24.24^{\star *}$ & 0.015 & $93.15 \pm 25.08$ & ns \\
\hline LIP & $91.54 \pm 12.22$ & ns & $115.92 \pm 13.60^{*}$ & 0.007 & $64.70 \pm 20.05^{\star *}$ & 0.000 \\
\hline TAU & $109.53 \pm 15.38$ & ns & $129.69 \pm 16.42$ & ns & $104.78 \pm 22.03$ & ns \\
\hline \multicolumn{7}{|l|}{ SOD $\mathrm{U} \cdot \mathrm{g}^{-1} \mathrm{Hb}$} \\
\hline CON & $1505 \pm 173$ & & $1089 \pm 188^{* *}$ & & $1238 \pm 163^{* *}$ & \\
\hline NAC & $1250 \pm 222$ & 0.000 & $1114 \pm 179$ & ns & $1243 \pm 119$ & ns \\
\hline LIP & $1600 \pm 166$ & ns & $1532 \pm 208$ & 0.000 & $1708 \pm 226$ & 0.000 \\
\hline TAU & $1351 \pm 162$ & 0.017 & $1225 \pm 182$ & ns & $1563 \pm 201^{*}$ & 0.000 \\
\hline \multicolumn{7}{|l|}{$\mathrm{GPx} U \cdot \mathrm{g}^{-1} \mathrm{Hb}$} \\
\hline CON & $51.83 \pm 5.33$ & & $45.63 \pm 7.10$ & & $42.65 \pm 3.57^{\star *}$ & \\
\hline NAC & $44.45 \pm 6.46$ & 0.028 & $51.46 \pm 8.86^{*}$ & 0.027 & $55.00 \pm 9.00^{* *}$ & 0.000 \\
\hline LIP & $34.16 \pm 8.80$ & 0.000 & $43.05 \pm 9.91^{*}$ & ns & $54.01 \pm 9.26^{* *}$ & 0.002 \\
\hline TAU & $42.49 \pm 8.52$ & 0.005 & $49.37 \pm 10.18^{*}$ & ns & $49.69 \pm 8.96^{*}$ & 0.033 \\
\hline \multicolumn{7}{|l|}{ CAT $\mathrm{k} \cdot \mathrm{g}^{-1} \mathrm{Hb}$} \\
\hline CON & $71.10 \pm 11.73$ & ns & $82.61 \pm 10.59^{*}$ & & $75.70 \pm 9.27$ & \\
\hline NAC & $77.03 \pm 7.80$ & ns & $81.57 \pm 9.26$ & ns & $81.38 \pm 8.99$ & ns \\
\hline LIP & $66.40 \pm 5.51$ & ns & $71.94 \pm 8.62$ & 0.035 & $71.47 \pm 8.81$ & ns \\
\hline TAU & $70.45 \pm 9.51$ & & $81.22 \pm 12.27^{*}$ & ns & $82.55 \pm 13.37$ & ns \\
\hline
\end{tabular}

${ }^{*} p<0.05$ and ${ }^{* *} p<0.01$ are significantly different compared with the pre-exercise value. Supp. vs. CON indicates statistically significant $(p<0.05)$ differences between the placebo group and the supplemented groups. ns indicates no statistically significant $(p>$ $0.05)$ differences between the placebo group and the supplemented groups. CON, placebo; NAC, N-acetylcysteine; LIP, $\alpha$-lipoic acid; TAU, taurine. 


\section{A. ZEMBRON-LACNY et al.}

Table 3. Relationship between the analyzed parameters in the CON group.

\begin{tabular}{lcc}
\hline & Coefficient of correlation & Significance \\
\hline SOD/TBARS & $r=-0.605$ & $0.002^{*}$ \\
CAT/TBARS & $r=0.412$ & $0.045^{*}$ \\
GPx/TBARS & $r=-0.218$ & 0.307 \\
${ }^{*} p<0.05$. &
\end{tabular}

TBARS concentration significantly increased more than $20 \%$ in response to exercise, and then declined to an initial level at $24 \mathrm{~h}$ rest. The correlations were observed between the activities of antioxidant enzymes and the intensity of peroxidation in the CON group (Table 3 ). At rest, the TBARS level demonstrated a tendency to lower its values after NAC and LIP. Immediately after exercise, peroxidation was markedly attenuated by NAC and LIP supplementation. Although TAU did not influence TBARS concentration compared with $\mathrm{CON}$, it seems that this supplement prevented an increase in peroxidation during exercise.

SOD activity decreased significantly by $28 \%$ at postexercise and by $18 \%$ at $24 \mathrm{~h}$ rest in the CON group. NAC and TAU administration caused a significant decrease in resting SOD activity, whereas LIP induced changes in the postexercise SOD level compared with CON. In the LIP group, a remarkable increase in enzyme activity, about $40 \%$, was observed. Even so, the SOD activity changed differently in response to the intake of cysteine deriva- tives; the level of SOD was higher in the NAC, LIP, and TAU groups than in the CON group, especially after exercise. This means that supplementation prevented an exercise-induced decline in SOD activity.

GPx activity significantly decreased by $18 \%$ at a $24 \mathrm{~h}$ rest in the CON group. The administration of cysteine derivatives significantly reduced resting GPx activity and also triggered an increase in enzyme activity after exercise. At $24 \mathrm{~h}$ of rest, the level of GPx increased by 17 $29 \%$ in supplemented groups compared with CON.

CAT activity increased by $16 \%$ immediately after exercise in the placebo group. NAC and TAU administration did not influence CAT activity, whereas LIP induced a $13 \%$ decrease in post-exercise CAT activity compared with CON.

The estimation of $\mathrm{P} / \mathrm{A}$ ratio showed the smallest disturbance of pro-antioxidant equilibrium after 3 days of LIP administration; i.e., $\alpha$-lipoic acid significantly decreased in $\mathrm{P} / \mathrm{A}$ ratio in contrast to the other applied compounds (Fig. 1). The $\mathrm{P} / \mathrm{A}$ ratio was positively correlated with plasma protein thiol concentration in CON (Fig. 2). The intake of cysteine derivatives abolished the observed relationship.

The number of performed series in applied resistance exercise or maximal loads was not significantly changed in subjects after supplementation with cysteine derivatives. The maximal load for the CON group was $92 \%$, for the NAC group $95 \%$, for the LIP group $94 \%$, and for the TAU group 91\% $1 \mathrm{RM}$.
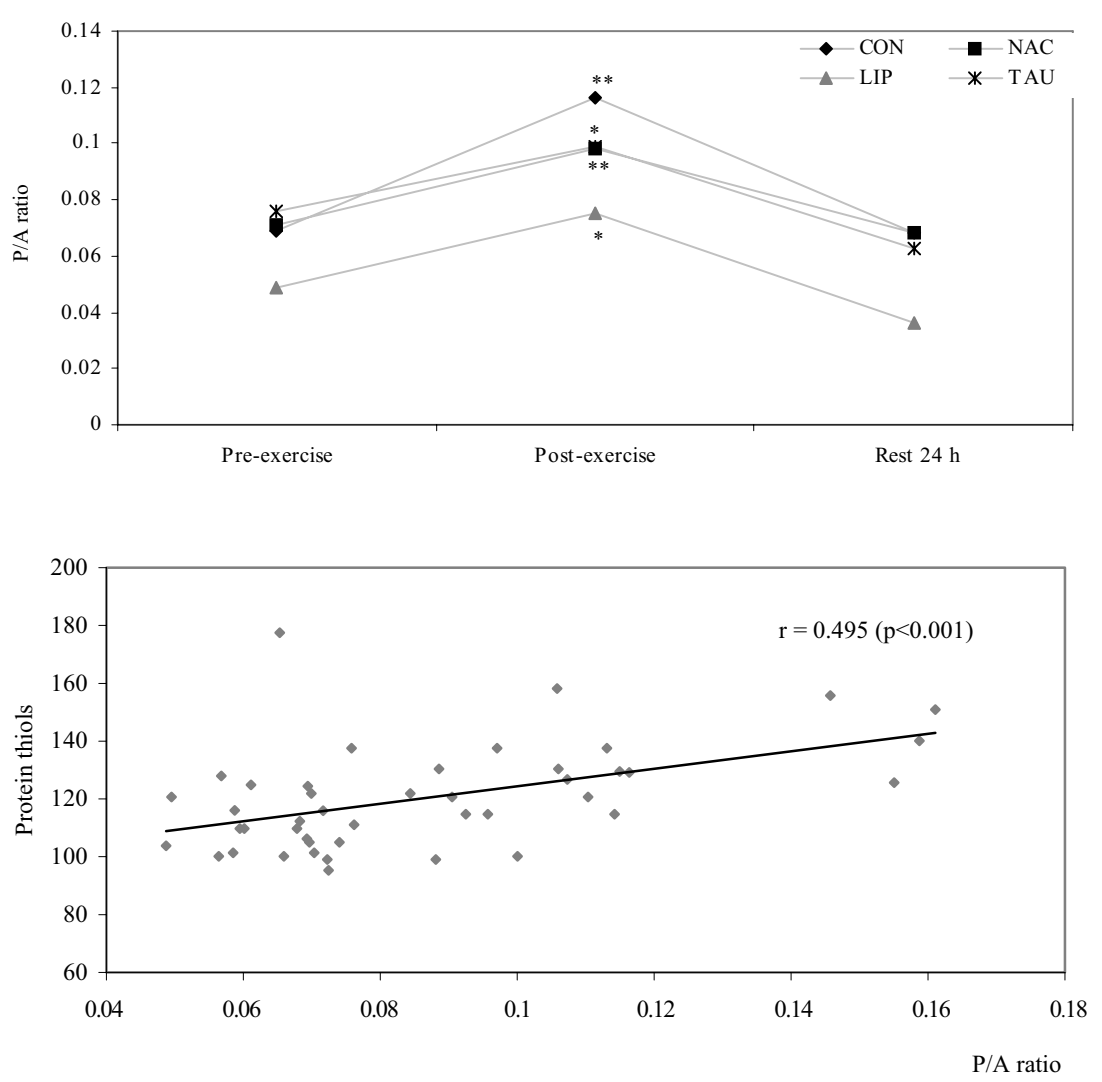

Fig. 1. Changes in P/A ratio. ${ }^{*} p<0.05$ and ${ }^{* *} p<0.01$ are significantly different compared to the pre-exercise value. Gray marks mean statistically significant different valus from the corresponding CON value.

Fig. 2. Relationship between the P/A ratio and the protein thiol level. 


\section{DISCUSSION}

The parameters of pro-antioxidant equilibrium such as antioxidant enzymes and peroxidation products play an important role in determining the risk of oxidative stress and the effectiveness of antioxidant supplementation. Unfortunately, the individual analysis of these parameters is complicated because of various changes in antioxidant enzyme activities and peroxidation markers in response to exercise. Therefore an attempt was made to simplify the study of pro-antioxidant equilibrium by using different formulas or methods. Klapcinska et al. [19] evaluated the index of "total antioxidant potential" (POTAOX), calculated as a sum of standardized values of individual antioxidant species. Earlier, Loverro et al. [18] described "proantioxidant coefficient" calculated from the mean values of TBARS, CAT, GPx, and SOD.

The additional cysteine derivatives intake by athletes in the present study increased in a resting concentration of plasma protein thiols in the NAC and LIP groups. This could have been caused by the increase of the main intraand extracellular thiol, i.e., glutathione. It was established that NAC is actively transported into cells, deacylated, and it increases cysteine availability for glutathione synthesis. Nielsen et al. [20] showed a high cysteine level in plasma after a 3-day NAC supplementation $\left(6 \mathrm{~g} \cdot \mathrm{day}^{-1}\right)$ in rowers. Medved et al. [21] observed that pre-exercise NAC infusion increased blood glutathione during sprint test and recovery. Also, our earlier study showed that NAC supplementation in athletes caused an increase of reduced glutathione (GSH) concentration in blood before and after strength exercise [22].

Lipoic acid could have increased in protein thiols concentration mainly by participation in disulfide glutathione (GSSG) reduction. Khanna et al. [5] observed an increase in GSH concentration at rest and after exhaustive treadmill exercise in rat liver and blood following intragastric lipoic acid supplementation. Busse et al. [4] added lipoic acid to mouse cell lines and observed a dose-dependent increase in GSH content by 30-70\% compared to untreated controls. These results were confirmed in human cell lines by the same authors, in which intracellular concentrations of GSH increased approximately by $50 \%$ in $5 \mathrm{~h}$ after the addition of lipoic acid to the culture medium.

Unlike NAC and LIP, a three-day supplementation of taurine led to no changes in protein thiol concentration. In fact the achieved result was contrary to the expected one. According to Dawson et al. [11] and Zhang et al. [12], taurine is a strong antioxidant that influences thiol concentration. Miyazaki et al. [23] showed that taurine administration in rats with 100 and $500 \mathrm{mg} \cdot \mathrm{kg}^{-1} \cdot \mathrm{d}^{-1}$ for 2 weeks affected glutathione level, inhibiting the oxidation glutathione. In our study, $3 \mathrm{~g} \cdot \mathrm{day}^{-1}$ taurine was applied for three days, which gave approximately $37 \mathrm{mg} \cdot \mathrm{kg}^{-1} \cdot \mathrm{day}^{-1}$. We suppose that the dose of taurine was insufficient or that the supplementation period was too short to elevate thiol level. Further, studies are necessary to explain this discrepancy and to establish the effective taurine dose.

The elevation of plasma protein thiol concentration by NAC and LIP administration was parallel with an attenuation of peroxidation following resistance exercise. The postexercise level of erythrocyte TBARS was lower in NAC and LIP compared with the TAU and CON groups. Unfortunately, TAU revealed no antioxidant action, i.e., peroxidation intensity did not change after taurine supplementation.

The applied resistance exercise caused a decrease in SOD activity. Our results are similar to findings of Tauler et al. [24] in athletes performing a long-term exercise and Vesovic et al. [25] in regard to a short-term exercise. According to Hodgson and Fridovich [26], the decrease in SOD activity is involved in irreversible enzyme inhibition by ROS. Despite the postexercise decrease in SOD activity, the level of SOD was elevated after a 24-h rest. Tauler et al. [23] explained the changes in antioxidant enzyme activities by the covalent modification of enzymes or some protein interactions. The other reason for an increase in SOD activity might be attributable to a release of erythrocyte "reservoirs," such as the liver or spleen containing unaffected SOD molecules [27].

The changes in SOD activity in response to used supplements were very different before and after exercise. Generally, it could be stated that the applied cysteine derivatives diminished the decrease in SOD activity induced by exercise. However, most changes were observed after $\alpha$-lipoic acid supplementation.

Another enzyme involved in pro-antioxidant equilibrium is the selenium enzyme GPx, removing hydrogen peroxide and lipid peroxides in the presence of glutathione as cofactor. Therefore ROS, lipid peroxidation, or the limitation of thiol availability affects mainly glutathione peroxidase (GPX) activity by allosteric or covalent modification. Previous studies showed either a decrease, increase, or no change in this enzyme's activity in blood after various physical exercises $[22,25,28]$. In the present study, GPx activity decreased upon exercise in CON, but not in the NAC, LIP, and TAU groups. On the other hand, GPx activity was significantly decreased in supplemented groups before exercise. We suppose that a 3-day intake of cysteine derivatives reduced ROS and peroxidation product levels and thus induced a decline in resting GPx activity. It should be stressed that LIP administration triggered the most decrease in GPx before exercise.

Contrary to SOD and GPx, erythrocyte CAT activity was significantly higher immediately after strength exercise. The increase of CAT activity following exercise was also observed by Marzatico et al. [29] and Wozniak et al. [30]. Erythrocytes are unable to synthesize proteins, so the recognized rapid rise of CAT activity could be related to some activation mechanism, similarly to SOD and GPx. 
The explanation of such response was offered by Tauler $e t$ al. [24], who suggested that high superoxide radical concentration leads to an elevation in CAT activity. The reason for the elevation of $\mathrm{O}_{2}{ }^{-}$level in erythrocytes is hemoglobin auto-oxidation and ROS diffusion through the erythrocyte membrane. Superoxide radical anions enter the red cells because of the presence of an anion exchanger (band 3) or as hydroperoxyl radicals formed in protonation reaction facilitated by the blood $\mathrm{pH}$ decrease during exercise [31].

As the above-mentioned information, the changes in pro-antioxidant equilibrium parameters make it difficult to state explicitly which cysteine derivative is the most effective antioxidant. The application of Loverro's coefficient [18] enables not only an analysis of pro-antioxidant equilibrium, but also an evaluation of antioxidant effectiveness in athletes.

The carried-out study showed first that the $\mathrm{P} / \mathrm{A}$ ratio directly correlated with plasma protein thiol concentration, which suggests a close relationship between pro-antioxidant equilibrium in erythrocyte and protein thiol content in plasma. This positive correlation also implies that a higher $\mathrm{P} / \mathrm{A}$ ratio leads to a more efficient defense against oxidative stress. Second, P/A ratio increased during exercise in each group, which indicates the enhancement of lipid peroxidation. At last, $\mathrm{P} / \mathrm{A}$ ratio significantly decreased only after a 3 -day intake of $\alpha$-lipoic acid compared with the placebo and other cysteine derivatives, which suggests that this supplement is the most effective. This could be related with a very low redox potential for dihydrolipoate/lipoic acid couple $(-0.32 \mathrm{~V})$ compared to other antioxidants. Therefore it can reduce glutathione, tocopherols, ascorbic acid, and also cystine [4].

Our results have shown that 3-day oral $\mathrm{N}$-acetylcysteine and $\alpha$-lipoic acid administration markedly influenced the parameters of pro-antioxidant equilibrium, whereas taurine showed lesser antioxidant action. The use of Lowerro's coefficient (1) demonstrated the practical application of the $\mathrm{P} / \mathrm{A}$ ratio to evaluate the effectiveness of antioxidants, (2) facilitated a comparison of the antioxidant effects of applied cysteine derivatives, and (3) confirmed the antioxidant effects of $\alpha$-lipoic acid in athletes.

\section{REFERENCES}

1. Dröge W. Free radicals in the physiological control of cell function. Physiol Rev. 2001;82:47-95.

2. Peake J, Suzuki K. Neutrophils, antioxidant supplements and exercise-induced oxidative stress. Exerc Immunol Rev. 2004;10:129-41.

3. Sen CK, Packer L. Thiol homeostasis and supplements in physical exercise. Am J Clin Nutr. 2000;72:653-69.

4. Packer L, Witt EH, Tritschler HJ. Alpha-lipoic acid as a biological antioxidant. Free Rad Biol Med. 1995;19:227-50.

5. Busse E, Zimmer G, Schopohl B, Kornhuber B. Influence of alpha-lipoic acid on intracellular glutathione in vitro and in vivo. Arzneimittelforschung. 1992;42:82931.

6. Khanna S, Atalay M, Laaksonen DE Gul M, Roy S, Sen CK. Lipoic acid supplementation: tissue glutathione homeostasis at rest and after exercise. J
Appl Physiol. 1999;86:1191-6.

7. Marsh SA, Laursen PB, Coombes JS. Effects of antioxidant supplementation and exercise training on erythrocyte antioxidant enzymes. Int J Vitamin Nutr Res. 2006;76:324-31.

8. Palaniappan AR, Dai A. Mitochondrial ageing and the beneficial role of $\alpha$-lipoic acid. Neurochem Res. 2007;32:1552-8.

9. Kinnunen S, Hyyppa S, Oksala N, Atalay M. Heat shock protein responses to exercise in standarbred trotters: effects of $\alpha$-lipoic acid. ECSS Jyvaskyla 2007 (abstract), http://www.jyu.fi/en/congress/ecss07/generalinfo.

10. Cuisinier C, Michotte de Welle J, Verbeck RK, Poortmans JR, Ward R, Sturbois $X$, Francaux M. Role of taurine in osmoregulation during endurance exercise. Eur J Appl Physiol. 2002;87:489-95.

11. Dawson R, Biasetti M, Messina S, Dominy J. The cytoprotective role of taurine in exercise-induced muscle injury. Amino Acids. 2002;22:309-24.

12. Zhang M, Izumi I, Kagamimori S, Sokejima S, Yamagami T, Liu Z, Qi B. Role of taurine supplementation to prevent exercise-induced oxidative stress in healthy young men. Amino Acids. 2004;26:203-7.

13. Hebeeb AFSA. Reaction of protein sulfhydryl groups with Ellman's reagent. Methods Enzymol. 1972;25:457-64.

14. Paglia DE, Valentine WN. Studies on the quantitative characterization of erythrocyte glutathione peroxidase. J Lab Clin Med. 1967;70:158-69.

15. Aebi H. Catalase. In: Rice-Evans CA, Diplock AT, Symons MCR, editors. Techniques in free radical research. Amsterdam, London, New York, Tokyo: Elsevier; 1991. p. 199-201.

16. Buege J, Aust SD. The thiobarbituric acid assay. In: Rice-Evans CA, Diplock AT, Symons MCR, eds. Techniques in free radical research. Amsterdam, London, New York, Tokyo: Elsevier; 1991. p. 47-148.

17. Drabkin DL. Spectrophotometric studies. XIV. The crystallographic and optical properties of the hemoglobin of man in comparison with those of other species. J Biol Chem. 1946;164:703-23.

18. Loverro G, Greco P, Capusano F, Carone G, Selvaggi L. Lipoperoxidation and antioxidant enzymes activity in pregnancy complicated with hypertension. Eur J Obstet Gynecol Reprod Biol 1996; 70:123-7.

19. Klapcinska B, Kempa K, Mossakowska M, Derejczyk J. Blood antioxidant potential as a predictor of "successful aging"? Results of a study on polish centenarians. International Congress Medicina Sportiva Krakow 2006, Med Sport. 2006;10:47 (abstract)

20. Nielsen HB, Kharazmi A, Bolbjerg ML, Poulsen HE, Pedersen BK, Secher NH. $\mathrm{N}$-acetylcysteine attenuates oxidative burst by neutrophils in response to ergometer rowing with no effect on pulmonary gas exchange. Int J Sports Med. 2001;22:256-60.

21. Medved I, Brown MJ, Bjorksten AR, Leppik JA, Sostaric S, McKenna MJ. Nacetylcysteine infusion alters blood redox status but not time to fatigue during intense exercise in humans. J Appl Physiol. 2003:94:1572-82.

22. Zembron-Lacny A, Szyszka K, Sobanska B. Effect of strength exercise and Nacetylcysteine on metabolism of glutathione, oxidative damage and erythropoietin production. Med Sport. 2005;9:33-9.

23. Miyazaki T, Matsuzaki Y, Ikegami T, Miyakawa S, Doy M, Tanaka M, Bouscarel B. Optimal and effective oral dose of taurine to prolong exercise performance in rat. Amino Acids. 2004;27:291-8.

24. Tauler P, Gimeno I, Aguilo A, Guix MP, Pons A. Regulation of erythrocyte antioxidant enzyme activities in athletes during competition and short-term recovery. Eur J Physiol. 1999;438:782-7.

25. Vesovic D, Borjanovic S, Markovic S, Vidakovic A. Strenuous exercise and action of antioxidant enzymes. Med Lav. 2002;93:540-50.

26. Hodgson EK, Fridovich I. The interaction of bovine erythrocyte superoxide dismutase with hydrogen peroxide: inactivation of the enzyme. Biochemistry. 1975; 14:5294-9.

27. Szygula Z. Erythrocytic system under the influence of physical exercise and training. Sports Med. 1990;10:181-97.

28. Cavas $L$. Does underwater rugby stimulate the over-production of reactive oxygen species? Cell Biochem Funct. 2004;23:59-63.

29. Marzatico F, Pansarasa O, Bertorelli L, Somenzini L, Della Valle G. Blood free radical antioxidant enzymes and lipid peroxides following long-distance and lactacidemic performances in highly trained aerobic and sprint athletes. J Sports Med Phys Fitness. 1997;37:235-9.

30. Wozniak A, Drewa G, Chesy G, Rakowski A, Rozwodowska M, Olszewska D. Effect of altitude on the peroxidation and antioxidant enzymes in sportsmen. Med Sci Sports Exerc. 2001;33:1109-13.

31. Mao GD, Poznansky MJ. Electron spin resonance study on the permeability of superoxide radicals in lipid bilayers and biological membranes. FEBS Lett. 1992;305:233-6. 